ARTÍCULO ORIGINAL

\title{
LUGAR DE RESIDENCIA Y GRADO DE MARGINACIÓN COMO FACTORES PRONÓSTICO DE SUPERVIVENCIA AL CÁNCER DE PRÓSTATA EN VERACRUZ, MÉXICO
}

\author{
Richy Rogelio Gutiérrez-Juárez (101,a, María Teresa Álvarez-Bañuelos (i] 1,b,

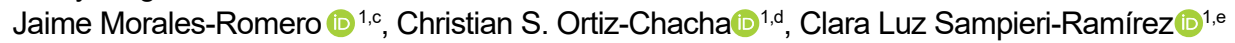 \\ 1 Instituto de Salud Pública, Universidad Veracruzana, Xalapa, Veracruz, México. \\ a Médico cirujano, ${ }^{\mathrm{b}}$ doctora en Ciencias Biológicas, ${ }^{\mathrm{c}}$ doctor en Ciencias de la Salud Pública, ${ }^{\mathrm{d}}$ doctor en Ciencias de la Salud,

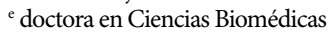

\section{RESUMEN}

Objetivos: Determinar si el lugar de residencia y el grado de marginación se encuentran asociados a la supervivencia al cáncer de próstata. Materiales y métodos: Se incluyeron a todos los pacientes diagnosticados con cáncer de próstata (CP) en el periodo 2013-2017 en un hospital de tercer nivel de atención de Veracruz, México. Los casos expuestos fueron los pacientes que habitualmente residían en zonas rurales, los no expuestos fueron los de zonas urbanas. Se recolectaron variables según características clínico epidemiológicas e histopatológicas. Para medir la supervivencia se utilizó el método de Kaplan Meier y la prueba de Log Rank. Los factores pronósticos fueron determinados calculando hazard ratio ajustado (HRa) en un análisis multivariado mediante el método de riesgos proporcionales de Cox. Resultados: Se analizaron 186 casos de CP. La supervivencia global a 5 años fue de 48,3\%. Los hombres que residían en zonas urbanas tuvieron una probabilidad de supervivencia mayor que quienes residían en zonas rurales (HRa 1,67; IC 95\%: 1,16-2,41). Asimismo, las personas que vivían en lugares catalogados como zonas de baja marginación tuvieron una mayor probabilidad de supervivencia que quienes vivían en zonas de alta marginación (HRa 2,32; IC 95\%: 1,47-3,66). Conclusiones: El lugar de residencia rural se identificó como un factor de mal pronóstico para la supervivencia de pacientes con CP independientemente de otras variables sociodemográficas y clínicas; los pacientes que vivían en lugares con grados de marginación más elevados tuvieron un pronóstico desfavorable de supervivencia.

Palabras clave: Cáncer de Próstata; Supervivencia; Población Rural; Factores Socioeconómicos; México (fuente: DeCS BIREME).

\section{PLACE OF RESIDENCE AND SOCIAL MARGINALIZATION AS PROGNOSTIC FACTORS FOR PROSTATE CANCER SURVIVAL IN VERACRUZ, MEXICO}

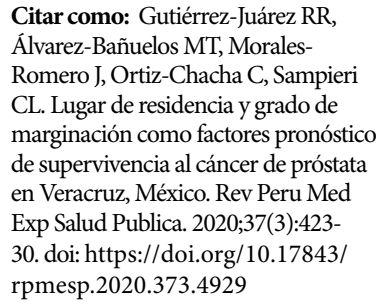

Correspondencia: María Teresa Álvarez-Bañuelos;talvarez@uv.mx

Recibido: $28 / 10 / 2019$ Aprobado: 03/06/2020 En línea: 03/08/2020

\section{ABSTRACT}

Objectives: To determine if the place of residence and the level of social marginalization are associated with prostate cancer survival. Materials and methods: All patients diagnosed with prostate cancer (PC) in the period from 2013 to 2017 in a tertiary healthcare hospital in Veracruz, Mexico were included. Patients resided in rural and urban areas. Variables were collected according to clinical-epidemiological and histopathological characteristics. The Kaplan Meier method and the Log Rank test were used to measure survival. Prognostic factors were determined by calculating the adjusted hazard ratio (HRa) in a multivariate analysis using the Cox proportional risk method. Results: A total of 186 PC cases were analyzed. Overall, after 5 years, $48.3 \%$ of the patients survived. Men living in urban areas had a higher probability of survival than those living in rural areas (HRa 1.67, 95\% CI 1.16-2.41). Similarly, people living in areas classified as low- marginalization zones had a higher probability of survival than those living in areas with a high level of social marginalization (HRa 2.32, 95\% CI 1.47-3.66). Conclusions: To reside in a rural place was identified as a negative prognostic factor for the survival of patients with PC regardless of other sociodemographic and clinical variables; patients living in high-marginalization places had an unfavorable survival prognosis

Keywords: Prostatic Neoplasms; Survival Analysis; Rural Population;Socioeconomic Factors; Mexico (Source: MeSH NLM). 


\section{INTRODUCCIÓN}

A nivel internacional, el cáncer de próstata (CP) es la segunda neoplasia con mayor incidencia en hombres (33,1 casos por cada 100000 personas) después del cáncer pulmonar, y es la quinta causa de muerte por neoplasias malignas en la población masculina ${ }^{(1)}$. En México, las defunciones por tumores malignos de próstata han mostrado un incremento en las últimas dos décadas ${ }^{(2)}$. El CP es causado por diversos factores, entre ellos destacan la edad, la dieta, la etnia, el color de piel, la exposición al tabaco o alcohol e incluso algunas infecciones ${ }^{(3,4)}$.

En México, el panorama del CP se caracteriza por una incidencia creciente, mayor frecuencia de presentación de los estadios avanzados, aumento de los costos de manejo y un incremento de la mortalidad ${ }^{(5,6)}$. Se ha documentado que la supervivencia del CP está relacionada con el estadio clínico al momento del diagnóstico y el tipo de tratamiento utilizado ${ }^{(5)}$. En este sentido, existe evidencia de una supervivencia a 5 años en diferentes tipos de cáncer, como pulmonar, hepático, colorrectal, mamario, pancreático, esofágico, vesical, y prostático ${ }^{(7)}$. Además, algunos estudios han demostrado una mayor supervivencia de los pacientes con CP que vivían en zonas urbanas y con un nivel socioeconómico más alto, comparado con los que procedían de zonas rurales y de bajo nivel socioeconómico ${ }^{(8,9)}$.

La asociación de tales características sobre la supervivencia ha sido poco estudiada en población mexicana, y la información en población veracruzana es casi nula. El objetivo de este estudio fue determinar si el lugar de residencia y el grado de marginación se encuentran asociadas a la supervivencia al cáncer de próstata en una cohorte hospitalaria.

\section{MATERIALES Y MÉTODOS}

\section{Diseño del estudio y participantes}

Se llevó a cabo un estudio de cohorte abierta retrospectivo en el que se incluyeron todos los pacientes diagnosticados con CP en el periodo 2013-2017 en el Centro Estatal de Cancerología (CECan), el cual es un hospital público de tercer nivel de atención ubicado en Xalapa, Veracruz, México. El periodo de seguimiento fue de 60 meses a partir del registro del primer caso de CP identificado durante el periodo de reclutamiento. Los criterios de inclusión fueron pacientes con diagnóstico de $\mathrm{CP}$ dentro del periodo de estudio y que recibieron atención en dicho hospital; los de exclusión, pacientes con antecedente de otro tipo de cáncer o que recibieron tratamiento antineoplásico previo; y los de eliminación, pacientes con diagnóstico de $\mathrm{CP}$ sin registros de datos de contacto para realizar el seguimiento.

\section{Variables}

La variable de respuesta principal fue el tiempo de supervivencia de los pacientes con $\mathrm{CP}$ definida como el tiempo

\section{MENSAJES CLAVE}

Motivación para realizar el estudio: No se han encontrado estudios recientes que evalúen la supervivencia al cáncer de próstata en México, por lo que se desconocen los factores asociados a esta medida.

Principales hallazgos: Se identificó una supervivencia al cáncer de próstata baja, además, se encontró que vivir en zonas rurales y con alto grado de marginación son factores de mal pronóstico para sobrevivir a esta enfermedad.

Implicancias: Se requieren más estudios que identifiquen los factores sociodemográficos e implicaciones asociados a este problema de salud pública, así como fortalecer las acciones sanitarias encaminadas a generar intervenciones que incrementen la supervivencia al cáncer de próstata.

transcurrido, en meses, entre la fecha del diagnóstico de $\mathrm{CP}$ y la defunción del paciente. El deceso se corroboró a través del certificado de defunción proporcionado por la coordinación de trabajo social del hospital y a través de los registros del Sistema Epidemiológico y Estadístico de Defunciones del sistema de salud estatal.

Las covariables sociodemográficas fueron escolaridad (con y sin estudios), grado de marginación de la localidad de procedencia (muy alto, alto, medio, bajo o muy bajo) de acuerdo con los índices establecidos por el Consejo Nacional de Población, el cual toma en cuenta ocho indicadores socioeconómicos (porcentaje de población de 15 años o más analfabeta, sin primaria completa, viviendas sin servicios de agua entubada, energía eléctrica, nevera, ni excusado, con piso de tierra y media de habitantes por habitación) ${ }^{(10)}$; y la zona de residencia habitual rural, si se trataba de lugares con población menor a 2500 habitantes, o urbana, si habitualmente vivían en lugares con más de 2500 habitantes, de acuerdo con la clasificación del Instituto Nacional de Estadística y Geografía (INEGI) ${ }^{(11)}$, otras covariables incluidas fueron ocupación (actividades agrícolas y afines vs otras actividades).

Las variables clínico-histopatológicas fueron comorbilidad (presencia o no de diabetes mellitus o hipertensión arterial), antígeno prostático específico (APE); $<10 \mathrm{ng} / \mathrm{dL} ; 10 \mathrm{a}$ $20 \mathrm{ng} / \mathrm{dL}$; > $20 \mathrm{ng} / \mathrm{dL}$, el grado de diferenciación histológica (bien diferenciado, moderadamente diferenciado y pobremente diferenciado), puntaje en la escala de Gleason ( $\leq 6,7 \mathrm{o}$ $\geq 8$ ), metástasis (presente o ausente), estadio clínico (I, II, III, o IV de acuerdo con los criterios del American Joint Committee on Cancer, AJCC). Etapa temprana (tumor localizado), etapa avanzada (el tumor se extiende a través de la cápsula prostática o invade estructuras adyacentes). Tipo de tratamiento: terapia hormonal neoadyuvante (antes de la prostatectomía) y la adyuvante (posterior a la prostatectomía) ${ }^{(12)}$. 
La supervivencia específica se calculó considerando el intervalo entre la fecha del diagnóstico histopatológico y la fecha de defunción o del término del seguimiento, lo que ocurriera primero.

\section{Instrumento de recolección de datos}

Se diseñó una cédula de acopio de datos que fue validada por tres médicos especialistas, tanto del hospital sede del estudio como del Instituto Nacional de Oncología y Radiobiología de La Habana, Cuba. La fuente de información fue el expediente clínico de los pacientes. La fecha y causa de defunción se obtuvo del certificado de defunción o de los registros del Sistema Epidemiológico y Estadístico de Defunciones (SEED).

\section{Análisis estadístico}

La comparación de las características demográficas y clínicas entre los estratos rural y urbano se realizó mediante la prueba chi-cuadrado o exacta de Fisher en variables categóricas, mientras que la comparación de la edad se realizó mediante la prueba t de Student. El análisis de supervivencia se calculó con el método de Kaplan-Meier; se consideraron como datos censurados a los pacientes que seguían vivos al término del seguimiento o cuyo estado actual era desconocido. Se compararon las probabilidades de supervivencia para cada posible factor pronóstico mediante la prueba de Log Rank, inicialmente especificando un modelo crudo para cada variable a analizar, y posteriormente se ajustaron modelos de riesgos proporcionales mediante regresión de Cox y se incluyeron variables de ajuste en el modelo, de acuerdo con la revisión de antecedentes sobre las variables pronósticas más importantes relacionadas con la supervivencia al cáncer de próstata ${ }^{(9,13,14)}$. En el análisis multivariado las covariables se dicotomizaron incluyendo el total de muestra: edad al momento del diagnóstico, lugar de residencia habitual, grado de marginación, estadio clínico, grado de diferenciación histológica y tipo de tratamiento. Se calcularon los hazard ratio (HR) crudo y ajustado y sus respectivos intervalos de confianza al 95\%. Los análisis se realizaron a través del software estadístico SPSS, versión 23.0 (IBM Inc., NY, EUA).

\section{Aspectos éticos}

El protocolo de investigación fue sometido y aprobado por el Consejo Técnico del Instituto de Salud Pública de la Universidad Veracruzana y por el Comité de Ética en Investigación del Centro Estatal de Cancerología "Dr. Miguel Dorantes Mesa”, con número de registro C.E.I.-2018-044.

\section{RESULTADOS}

\section{Características generales}

Se identificaron 198 pacientes con CP, de los cuales se excluyeron 12 casos que no cumplían los criterios de inclusión.
Finalmente, se analizaron los expedientes de 186 pacientes. La letalidad fue de $28,5 \%(\mathrm{n}=53)$. Se perdió el seguimiento del 8,6\% ( $\mathrm{n}=16)$. La media de edad al momento del diagnóstico fue de 69,2 $\pm 8,96$ años, y la media de la edad al momento del fallecimiento fue de 76,3 .

\section{Diferencias entre el grupo urbano y rural}

El 62,4\% de los pacientes vivían en zonas rurales. Las diferencias fueron estadísticamente significativas al comparar las proporciones de los grupos de zona rural y zona urbana por escolaridad, grado de marginación, ocupación y presencia de metástasis (Tabla 1).

Tabla 1. Características sociodemográficas y clínico-patológicas de la cohorte según el lugar de residencia

\begin{tabular}{|c|c|c|c|}
\hline \multirow{3}{*}{ Variable } & \multicolumn{2}{|c|}{ Lugar de residencia } & \multirow{3}{*}{$\begin{array}{l}\text { Valor } \\
\text { de p }\end{array}$} \\
\hline & Urbano & Rural & \\
\hline & $\mathrm{n}=\mathbf{7 0}(\%)$ & $\mathrm{n}=116(\%)$ & \\
\hline \multicolumn{4}{|l|}{ Edad } \\
\hline Media (DE) & $68( \pm 8,4)$ & $70( \pm 9,2)$ & $0,100^{\mathrm{b}}$ \\
\hline \multicolumn{4}{|l|}{ Escolaridad } \\
\hline Sin estudios & $19(27,1)$ & $52(44,8)$ & \multirow{2}{*}{$0,016^{\mathrm{a}}$} \\
\hline Con estudios & $51(72,9)$ & $64(55,2)$ & \\
\hline \multicolumn{4}{|l|}{$\begin{array}{l}\text { Grado de marginación } \\
\text { socioeconómica }\end{array}$} \\
\hline Muy bajo-Bajo & $25(35,7)$ & $18(20,5)$ & \multirow{3}{*}{$0,002^{\mathrm{a}}$} \\
\hline Medio & $31(44,3)$ & $53(60,2)$ & \\
\hline Alto-Muy alto & $14(20,0)$ & $17(19,3)$ & \\
\hline \multicolumn{4}{|l|}{ Ocupación } \\
\hline Agrícolas y afines & $47(67,1)$ & $104(89,7)$ & \multirow{2}{*}{$<0,00^{\text {a }}$} \\
\hline Otros & $23(32,9)$ & $12(10,3)$ & \\
\hline \multicolumn{4}{|l|}{$\begin{array}{l}\text { Comorbilidad } \\
\text { (diabetes o hipertensión) }\end{array}$} \\
\hline Sí & $16(22,9)$ & $34(29,3)$ & \multirow[t]{2}{*}{$0,336^{\mathrm{a}}$} \\
\hline No & $54(77,1)$ & $82(70,7)$ & \\
\hline \multicolumn{4}{|l|}{ Puntaje en escala de Gleason } \\
\hline$\leq 6$ & $29(41,4)$ & $60(51,7)$ & \multirow{3}{*}{$0,073^{\mathrm{a}}$} \\
\hline 7 & $19(27,1)$ & $16(13,8)$ & \\
\hline$\geq 8$ & $22(31,4)$ & $40(34,5)$ & \\
\hline \multicolumn{4}{|l|}{ Estadio clínico } \\
\hline I & $3(4,3)$ & $7(6,0)$ & \multirow{4}{*}{$0,057^{\mathrm{c}}$} \\
\hline II & $19(27,1)$ & $30(25,9)$ & \\
\hline III & $36(51,4)$ & $40(34,5)$ & \\
\hline IV & $12(17,1)$ & $39(33,6)$ & \\
\hline \multicolumn{4}{|l|}{ Grado histológico } \\
\hline Bien diferenciado & $7(10,0)$ & $8(6,9)$ & \multirow{3}{*}{$0,726^{\mathrm{a}}$} \\
\hline $\begin{array}{l}\text { Moderadamente } \\
\text { diferenciado }\end{array}$ & $27(38,6)$ & $44(32,9)$ & \\
\hline Pobremente diferenciado & $36(51,4)$ & $64(55,2)$ & \\
\hline \multicolumn{4}{|l|}{ Presencia de metástasis } \\
\hline Positiva & $12(17,1)$ & $39(33,6)$ & \multirow[t]{2}{*}{$0,023^{\mathrm{a}}$} \\
\hline Negativa & $58(82,9)$ & $77(66,4)$ & \\
\hline \multicolumn{4}{|l|}{ Localización de metástasis } \\
\hline Ósea & $8(75,0)$ & $28(71,8)$ & \multirow[t]{2}{*}{$0,054^{\mathrm{a}}$} \\
\hline Otra & $4(25,0)$ & $11(28,2)$ & \\
\hline
\end{tabular}

${ }^{\mathrm{a}}$ prueba Chi cuadrado, ${ }^{\mathrm{b}}$ prueba t de Student, ${ }^{\mathrm{c}}$ prueba exacta de Fisher. 
Al comparar las características clínico patológicas, el estadio III fue el más frecuente en ambas zonas, a diferencia, el estadio IV se presentó 3,25 veces menos en los pacientes con residencia habitual urbana en comparación con los de zona rural. De la misma manera, las metástasis óseas fueron 3 veces más frecuentes en los de zona urbana que en la rural (Tabla 1).

\section{Análisis de sobrevida}

La supervivencia global a un año fue de 70,6\% (IC 95\%: 62,1 a 77,5) para los sujetos de la cohorte de 2013. La supervivencia global a 3 años fue de 70,6\% (IC 95\%: 62,1 a 77,5), mientras que a 5 años fue de 47,7\% (IC 95\%: 31,7 a 63,0).
Se observaron diferencias estadísticamente significativas en la supervivencia según grado de marginación, donde los pacientes con alto/muy alto grado de marginación presentaron menor supervivencia que aquéllos procedentes de áreas de marginación muy bajo o bajo. Asimismo, la sobrevida de los pacientes procedentes de zonas de marginación media fue incluso mayor que la de aquellos de marginación baja/muy baja (Tabla 2).

Según el estadio clínico se observó una supervivencia menor en los sujetos en etapa IV comparado con los estadios I y II. De acuerdo con el grado de diferenciación histológica (Figura 1), la proporción acumulada que sobrevivió al final del intervalo de 60 meses fue $73 \%$ del grupo bien diferencia-

Tabla 2. Supervivencia a 1,3 y 5 años de seguimiento de los sujetos de la cohorte

\begin{tabular}{|c|c|c|c|c|c|}
\hline \multirow{3}{*}{ Variable } & \multirow{3}{*}{ Pacientes vivos } & \multicolumn{3}{|c|}{ Supervivencia $^{\text {a }}$} & \multirow{3}{*}{ Valor de $p^{b}$} \\
\hline & & 1 año & 3 años & 5 años & \\
\hline & & n (\%) & n (\%) & n (\%) & \\
\hline \multicolumn{6}{|l|}{ Edad (años) } \\
\hline$\leq 60$ & 35 & $27(76)$ & $16(60)$ & $4(25)$ & \multirow{2}{*}{0,051} \\
\hline$>60$ & 151 & $131(87)$ & $96(73)$ & $54(56)$ & \\
\hline \multicolumn{6}{|l|}{ Lugar de residencia } \\
\hline Rural & 116 & $93(80)$ & $56(60)$ & $54(28)$ & \multirow{2}{*}{$<0,001$} \\
\hline Urbano & 70 & $64(91)$ & $49(77)$ & $35(71)$ & \\
\hline \multicolumn{6}{|l|}{ Escolaridad } \\
\hline Sin estudios & 71 & $58(81)$ & $35(61)$ & $16(45)$ & \multirow{2}{*}{0,118} \\
\hline Con estudios & 115 & $100(87)$ & $76(76)$ & $37(49)$ & \\
\hline \multicolumn{6}{|l|}{ Ocupación } \\
\hline Agrícolas y afines & 151 & $124(82)$ & $88(71)$ & $45(51)$ & \multirow{2}{*}{0,289} \\
\hline Otros & 35 & $33(94)$ & $22(68)$ & $7(32)$ & \\
\hline \multicolumn{6}{|c|}{ Grado de marginación socioeconómica } \\
\hline Muy bajo-Bajo & 43 & $38(89)$ & $26(69)$ & $18(69)$ & \multirow{3}{*}{$<0,001$} \\
\hline Medio & 84 & $80(95)$ & $73(91)$ & $59(81)$ & \\
\hline Alto-Muy alto & 59 & $41(69)$ & $20(48)$ & $4(18)$ & \\
\hline \multicolumn{6}{|l|}{ Estadio clínico } \\
\hline I & 10 & $10(100)$ & $10(100)$ & $9(86)$ & \multirow[t]{4}{*}{$<0,001$} \\
\hline II & 49 & $46(94)$ & $39(85)$ & $30(77)$ & \\
\hline III & 76 & $67(88)$ & $47(71)$ & $20(43)$ & \\
\hline IV & 51 & $34(66)$ & $15(46)$ & $4(29)$ & \\
\hline \multicolumn{6}{|l|}{ Gleason } \\
\hline Riesgo bajo & 15 & $15(100)$ & $14(92)$ & $10(76)$ & \multirow[t]{3}{*}{0,152} \\
\hline Riesgo moderado & 75 & $63(84)$ & $47(74)$ & $31(67)$ & \\
\hline Riesgo alto & 96 & $79(82)$ & $50(64)$ & $7(13)$ & \\
\hline \multicolumn{6}{|l|}{ Metástasis } \\
\hline Presente & 51 & $34(66)$ & $15(46)$ & $4(29)$ & \multirow{2}{*}{$<0,001$} \\
\hline Ausente & 135 & $123(91)$ & $97(79)$ & $52(54)$ & \\
\hline \multicolumn{6}{|c|}{ Localización de metástasis } \\
\hline Óseas & 40 & $27(68)$ & $12(44)$ & $3(28)$ & \multirow{2}{*}{0,955} \\
\hline Otras & 11 & $6(58)$ & $4(58)$ & $1(29)$ & \\
\hline \multicolumn{6}{|l|}{ Tipo de tratamiento } \\
\hline Adyuvante & 93 & $83(89)$ & $64(77)$ & $28(44)$ & \multirow{2}{*}{0,031} \\
\hline Neoadyuvante & 93 & $73(79)$ & $46(63)$ & $21(46)$ & \\
\hline
\end{tabular}

${ }^{\text {a }}$ Método de Kaplan-Meier, ${ }^{\mathbf{b}}$ prueba de Log Rank. 


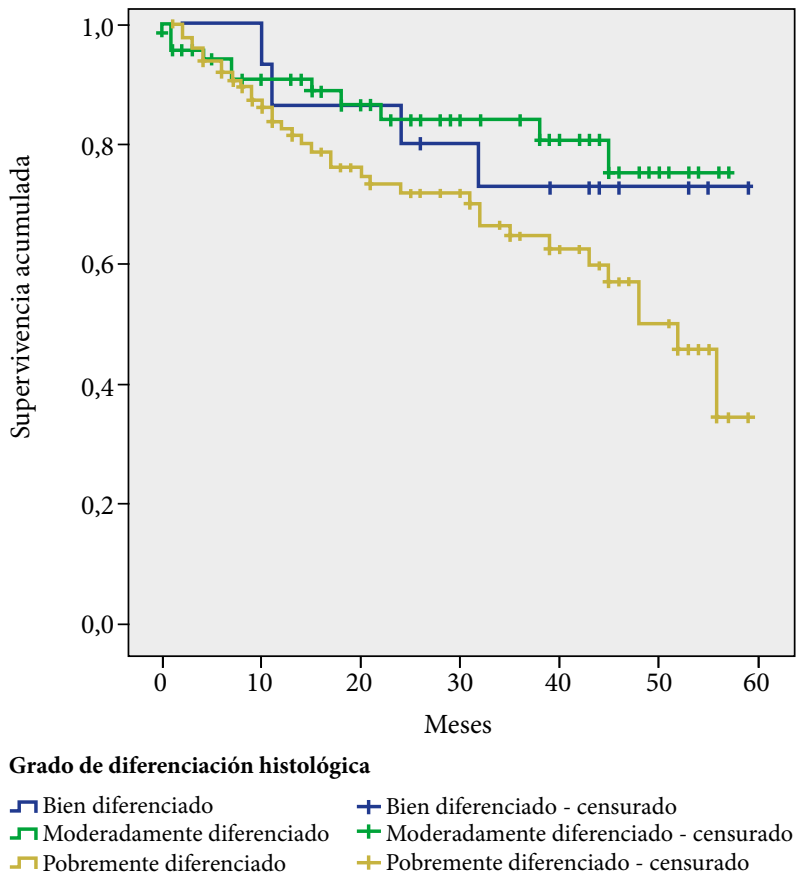

Figura 1. Curva de supervivencia acumulada de cáncer de próstata según grado de diferenciación histológica

do, $70 \%$ de los moderadamente diferenciados y $30 \%$ en los probremente diferenciados $(\mathrm{p}=0,035)$.

Se observó una menor supervivencia entre los sujetos con niveles séricos de antígeno prostático específico (APE) mayores de $20 \mathrm{ng} / \mathrm{dL}$ previo al tratamiento en comparación con aquellos con niveles de 10 a 20 y $<10$ ng/dL (Figura 2);

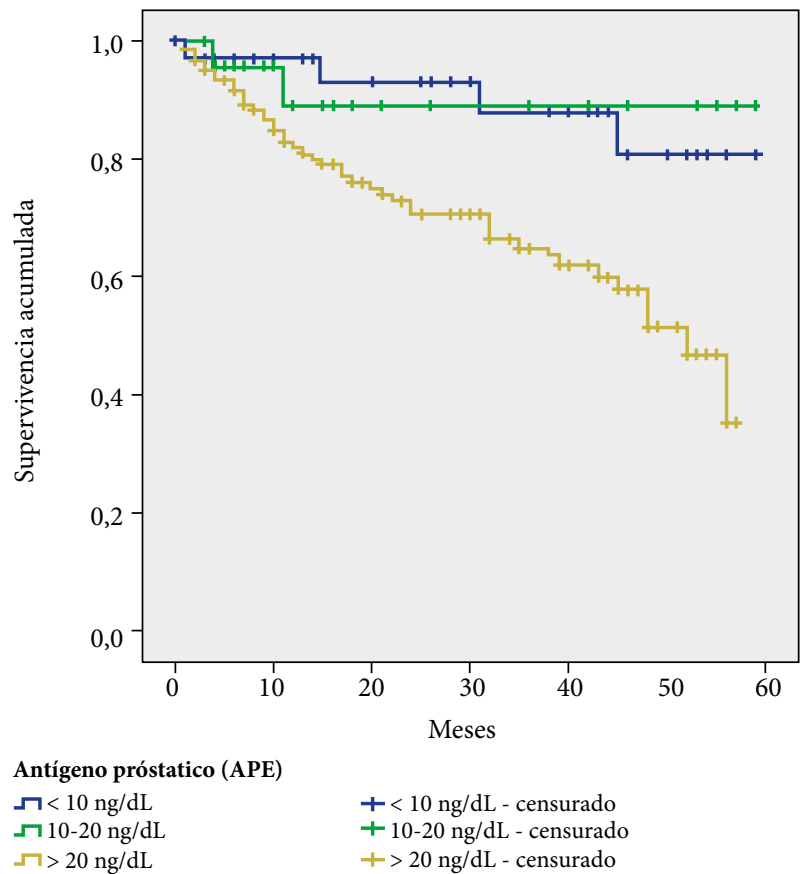

Figura 2. Curva de supervivencia acumulada cáncer de próstata según niveles de antígeno prostático las probabilidades de supervivencia a 5 años fueron de $31 \%$, $83 \%$ y $84 \%$, respectivamente $(\mathrm{p}=0,003$ ).

En la Tabla 3 se presenta un análisis multivariado de posibles predictores de supervivencia en pacientes con cáncer de próstata. En el modelo ajustado, las características de la residencia habitual como el tipo urbano (HRa: 1,67, IC 95\%: 1,16 a 2,41) y un bajo grado de marginación (HRa: 2,32 , IC 95\%: 1,47 a 3,66) mostraron una mayor probabilidad de supervivencia que aquellos provenientes de una localidad de tipo rural o con alto grado de marginación. No se encontraron diferencias significativas en la probabilidad de supervivencia con respecto al estadio clínico, la edad al momento del diagnóstico, la diferenciación histológica y el tipo de tratamiento.

\section{DISCUSIÓN}

Los resultados encontrados muestran una edad media al momento del diagnóstico de 69 años similar a la reportada en otros estudios previos realizados en México ${ }^{(15)}$, Brasil ${ }^{(16)}$ y Colombia ${ }^{(17)}$, en los cuales se hallaron medias de edad y desviación estándar de 66,7 $( \pm 8,8), 70,5( \pm 8,7)$ y $69( \pm 8,6)$ años,, respectivamente. La letalidad fue de $28 \%$, similar al $24 \%$ reportado por Silveira et al. en 2013 en un hospital brasileño ${ }^{(18)}$

Cerca del $68 \%$ de los casos fueron diagnosticados en etapas avanzadas (estadio III y IV), cifra superior a la reportada en otro estudio realizado en población mexicana ${ }^{(15)}$, en el cual se reportó solo el 46,2\% de casos en las mismas etapas. Las diferencias podrían explicarse dado que cada uno de los hospitales en donde se realizaron los estudios atiende a población con diferentes características sociodemográficas, principalmente en cuanto a su lugar habitual de residencia, ocupación y escolaridad.

Cabe mencionar que se ha encontrado que en algunos países como Estados Unidos y Nueva Zelanda las personas que viven en zonas rurales o menos aventajadas tienen una menor probabilidad de supervivencia, menor acceso a los servicios de salud, mayor mortalidad, menores tasas de realización de pruebas de APE y mayor riesgo de CP avanzado ${ }^{(8)}$. En cuanto a lo anterior, es necesario precisar que la ubicación geográfica provee información relacionada con la composición poblacional y el acceso a ciertos recursos y servicios, entre otros aspectos; mientras que el grado de aventajamiento refleja la probable influencia de la comunidad y la estructura social sobre las el riesgo de desarrollar la enfermedad, independientemente de su nivel socioeconómico individual ${ }^{(8)}$. Sobre la supervivencia según lugar de residencia habitual, algunos estudios han mostrado una menor sobrevida en personas de residencia rural con cáncer de próstata ${ }^{(7,14,19)}$.

Es esencial destacar que, entre los hallazgos de este estudio, se observó una supervivencia específica al CP a 5 años cercana al $48 \%$, cifra que equivale a casi la mitad de lo re- 
Tabla 3. Predictores de supervivencia en pacientes con cáncer de próstata

\begin{tabular}{|c|c|c|c|c|}
\hline \multirow{2}{*}{ Variable } & Modelo crudo & \multirow{2}{*}{ Valor de p } & Modelo multivariado & \multirow{2}{*}{ Valor de p } \\
\hline & HR (IC 95\%) & & HR (IC 95\%) & \\
\hline \multicolumn{5}{|l|}{ Lugar de residencia habitual } \\
\hline Rural & 1,00 & \multirow{2}{*}{0,013} & 1,00 & \multirow{2}{*}{0,006} \\
\hline Urbano & $1,56(1,10-2,22)$ & & $1,67(1,16-2,41)$ & \\
\hline \multicolumn{5}{|c|}{ Grado de marginación socioeconómica } \\
\hline Alto & 1,00 & \multirow{2}{*}{$<0,001$} & 1,00 & \multirow{2}{*}{$<0,001$} \\
\hline Bajo & $2,29(1,46-3,60)$ & & $2,32(1,47-3,66)$ & \\
\hline \multicolumn{5}{|l|}{ Estadio clínico } \\
\hline Avanzado & 1,00 & \multirow{2}{*}{0,359} & 1,00 & \multirow{2}{*}{0,443} \\
\hline Temprano & $1,18(0,83-1,68)$ & & $1,15(0,80-1,66)$ & \\
\hline \multicolumn{5}{|c|}{ Edad al momento del diagnóstico (años) } \\
\hline$>60$ & 1,00 & \multirow{2}{*}{0,306} & 1,00 & \multirow{2}{*}{0,942} \\
\hline$\leq 60$ & $1,28(0,80-2,05)$ & & $1,02(0,63-1,66)$ & \\
\hline \multicolumn{5}{|c|}{ Grado de diferenciación histológica } \\
\hline Escasamente diferenciado & 1,00 & \multirow{2}{*}{0,427} & 1,00 & \multirow{2}{*}{0,168} \\
\hline Bien diferenciado & $1,15(0,82-1,62)$ & & $1,29(0,90-1,84)$ & \\
\hline \multicolumn{5}{|l|}{ Tipo de tratamiento } \\
\hline Adyuvante & 1,00 & \multirow{2}{*}{0,909} & 1,00 & \multirow{2}{*}{0,956} \\
\hline Neoadyuvante & $0,98(0,70-1,39)$ & & $1,01(0,71-1,43)$ & \\
\hline
\end{tabular}

HR: Hazard ratio; IC95\%: Intervalo de confianza al 95\%.

portado por otros países latinoamericanos incluidos en el más amplio estudio realizado a nivel internacional (CONCORD-3) ${ }^{(20)}$, en el cual se reportan cifras superiores al $80 \%$ en algunos casos como el de Brasil, Costa Rica, Argentina, Ecuador y Uruguay; mientras que en la población colombiana según Arias y De Vries ${ }^{(21)}$ dicha probabilidad se encuentra alrededor de 70 \% (IC 95\%: 65,3 a 76,1).

Se debe aclarar que, por un lado, en dichos estudios se analizaron datos con base poblacional a diferencia de este trabajo en el que se recopilaron datos de base hospitalaria; por otro lado, los pacientes incluidos contaban con un mismo régimen de aseguramiento (Seguro Popular), procedían en mayor proporción de zonas rurales $(62,4 \%)$ y requerían trasladarse desde su lugar de residencia hasta el CECan, ubicado en la capital del Estado de Veracruz, por lo que dichos pacientes pueden enfrentar barreras de tipo geográfico y económico principalmente, así como tiempos de espera prolongados para el diagnóstico y tratamiento, lo cual puede limitar o retrasar su acceso a la atención médica ${ }^{(22,23)}$ y, en consecuencia, disminuir su probabilidad de supervivencia.

En contraste con lo anterior, cuando se comparó la probabilidad de supervivencia a 1, 3 y 5 años $(84,6 \%, 70,6 \%$, $47,7 \%$, respectivamente) con estudios de base hospitalaria, los hallazgos del presente estudio fueron similares a las encontradas en otra investigación realizada en Irán, en el que se reportaron cifras de $87 \%, 73 \%$, y $54 \%$, respectivamente ${ }^{(24)}$.

En relación con la supervivencia a 5 años en los pacientes en estadio I (86\%), esta fue similar a la reportada por
Migowsky et al ${ }^{(16)}$ en un estudio realizado en Brasil con una muestra de 258 pacientes, en la que dicho valor fue de $87,8 \%$ (IC 95\%: 83,3 a 92,5).

Los resultados de este estudio proporcionan argumentos para decir que en una alta proporción de pacientes en estadio avanzado (68,3\%), la supervivencia disminuyó a medida que el estadio incrementó, como se puede ver en el estadio IV en el que la supervivencia (29\%) fue casi la mitad de lo alcanzado en otros trabajos en los que se reportaron cifras entre $40 \%$ y $60 \%{ }^{(17,18,24)}$. Estos datos denotan que la identificación de casos o la búsqueda de atención se presentan de manera tardía, disminuyendo la probabilidad de sobrevivir, lo que sugiere la necesidad de incrementar los esfuerzos para la detección en estadios tempranos ${ }^{(18)}$.

En cuanto a la supervivencia según lugar de residencia habitual, algunos estudios han mostrado una menor sobrevida en personas de residencia rural con $\mathrm{CP}{ }^{(7,8,19)}$. Li et al. ${ }^{(7)}$ encontraron que la supervivencia a 5 años a diferentes tipos de cáncer, entre los que se encontraban el pulmonar, hepático, colorrectal, mamario, pancreático, esofágico, vesical, y prostático, era mayor en los pacientes de zonas urbanas comparado con los de zonas rurales (44,05 vs. $41,47 \%$, $\mathrm{p}<0,001)$; mientras que, respecto al CP específicamente, esta cifra también fue mayor en zonas urbanas con diferencia estadísticamente significativa (59,2\% vs. 53,3\%, p = 0,02).

Existe evidencia de variaciones en la supervivencia entre las diferentes áreas geográficas, demostrada principalmente en estudios que comparan pacientes con cáncer de zonas 
rurales y urbanas. Dichas variaciones pueden estar relacionadas con la exposición a factores de riesgo, así como como con las condiciones diferenciales para el acceso a los servicios de salud y por lo tanto a las pruebas de detección temprana y al tratamiento oportuno del cáncer ${ }^{(7,25,26)}$.

En ese sentido, en un meta-análisis realizado en 2015 por Baade et al. ${ }^{(8)}$ que incluyó trabajos de diferentes países de altos ingresos, se observó que la razón de riesgo de supervivencia fue casi 1,7 veces mayor en pacientes que vivían en zonas urbanas comparado con los de zonas rurales.

Otro de los hallazgos del estudio fueron las diferencias encontradas en cuanto al grado de marginación, en el modelo ajustado, los pacientes con un bajo grado de marginación mostraron mayor supervivencia comparado con los de alto grado de marginación. Resultados similares en estudios previos demostraron una asociación entre un nivel socioeconómico alto y una mayor probabilidad de supervivencia como se puede observar en el análisis de Bravo et al. ${ }^{(27)}$ donde los hombres diagnosticados con CP que vivían en zonas más aventajadas tenían mejor supervivencia que los de zonas menos aventajadas, independientemente del estadio clínico al momento del diagnóstico (HR: 3,5, IC 95\% 2,37 a 5,40).

Los estudios analizados confirman la asociación entre el grado de deprivación o desventaja socioeconómica y la supervivencia al $\mathrm{CP}$ en otros países. En todos ellos se ha observado que la supervivencia es menor en los hombres con mayor deprivación socioeconómica comparado con los de niveles socioeconómicos más alto o más aventajados ${ }^{(14,21,28-30)}$.

En el caso de México, en los Estados con mayor marginación se observa una menor cantidad de recursos tecnológicos y humanos para la atención de los problemas de salud; sin embargo, se han encontrado factores económicos, geográficos, educativos y culturales que pueden estar relacionados con la reducción en el diagnóstico y el tratamiento oportuno, lo cual puede influir sobre las cifras de supervi- vencia al CP en las personas que viven en lugares con dichas características y que apoyan los resultados encontrados en este estudio ${ }^{(23)}$.

Las principales limitaciones de este estudio incluyen su naturaleza retrospectiva y las restricciones inherentes a la calidad de la información de los expedientes clínicos, en los cuales se identificó en escasa proporción el estadio de los pacientes al momento del diagnóstico, por lo que al discernir tal etapa a partir de los demás datos clínicos podrían haberse generado errores de clasificación. Además, el grado de marginación de cada caso se estableció mediante el uso de los catálogos elaborados en cierto momento por zona geográfica, por lo que este dato podría no reflejar en la totalidad de los casos la realidad individual de los pacientes.

En conclusión, el lugar de residencia rural se identificó como un factor de mal pronóstico para la supervivencia de pacientes con $\mathrm{CP}$ independientemente de otras variables sociodemográficas y clínicas. Asimismo, los pacientes que vivían en lugares con grados de marginación más elevados tuvieron un pronóstico desfavorable de supervivencia. Las diferencias en la supervivencia por características sociodemográficas evidencian la necesidad de disminuir las brechas de desigualdad entre personas que residen en ámbitos rurales y con mayores grados de marginación.

Contribución de los autores: GJRR participó en el diseño, recolección de datos, análisis e interpretación de los datos y redacción del manuscrito. ABMT participó en la concepción, diseño, análisis e interpretación de datos, así como en redacción y la revisión del artículo. MRJ, OCCS, y SRCL participaron en el análisis e interpretación de los datos, y en la revisión del manuscrito. Todos los autores asumen la responsabilidad de cada uno de los apartados, además declaran haber revisado el contenido y aprobado la versión final del artículo.

Financiamiento: Autofinanciado.

Conflicto de interés: Los autores declaran no tener conflictos de interés.

\section{REFERENCIAS BIBLIOGRÁFICAS}

1. IARC/OMS. Global observatory of cancer [Internet]. GLOBOCAN 2018. 2018 [citado el 10 de mayo de 2018]. Disponible en: http:// globocan.iarc.fr/Pages/fact_sheets_population.aspx.

2. Instituto Nacional de Estadística y Geografía. Principales causas de mortalidad por residencia habitual, grupos de edad y sexo del fallecido. [Internet]. 2017 [citado el 2 de diciembre de 2017]. Disponible en: http://www.inegi.org.mx/est/contenidos/proyectos/registros/vitales/ mortalidad/tabulados/ConsultaMortalidad.asp.

3. Ferrís-Tortajada J, García-Castell J, Berbel -Tornero O, Ortega- García JA. Constitutional risk factors in prostate cancer. Actas Urol Esp. 2011;35(5):282-8. doi: 10.1016/j.acuroe.2011.06.005.

4. Bashir MN. Epidemiology of prostate cancer. Asian Pac J Cancer Prev. 2015;16(13):5137-41. doi: 10.1016/j.acuroe.2011.06.005.

5. Torres-Sánchez LE, Espinoza-Giacinto R, Rojas-Martínez R, Escamilla-Nuñez C, Vázquez-Salas RA, Campuzano JC, et al. Prostate cancer mortality according to marginalization status in Mexican states from 1980 to 2013. Salud Publica Mex. 2016;58(2):179-86. doi: 10.21149/ spm.v58i2.7787.
6. Mohar-Betancourt A, Reynoso-Noveron N, Armas-Texta D, Gutierrez-Delgado C, Torres-Dominguez JA. Cancer trends in Mexico : Essential data for the creation and follow-up of public policies. Am Soc Clin Oncol. 2017;3(6):740-8. doi: 10.1200/JGO.2016.007476.

7. Li X, Deng Y, Tang W, Sun Q, Chen Y, Yang C. Urban-Rural disparity in cancer incidence, mortality, and survivals in Shanghai, China, during 2002 and 2015. Fontiers Oncol. 2018;8(3):71-80. doi: 10.3389/ fonc.2018.00579.

8. Baade PD, Yu XQ, Smith DP, Dunn J, Chambers SK. Geographic disparities in prostate cancer outcomes - Review of international patterns. Asian Pacific J Cancer Prev. 2015;16(3):1259-75. doi: 10.7314/APJCP.2015.16.3.1259.

9. Rapiti E, Fioretta G, Schaffar R, Neyroud-caspar I. Impact of Socioeconomic Status on Prostate Cancer Diagnosis, Treatment, and Prognosis. Cancer. 2009;115(23):5556-65. doi: 10.1002/cncr.24607.

10. Consejo Nacional de Población. Metodología de estimación del índice de marginación por localidad [Internet]. 2010. Disponible en: http:// www.conapo.gob.mx/es/CONAPO/Indice_de_Marginacion_por_Localidad_2010. Published 2010. 
11. Teresa M, Macgregor G De. Desarrollo y distribución de la población urbana en México. Investig Geogr. 2003;(50):77-91.

12. Secretaria de Salud. Diagnóstico y Tratamiento del Cáncer de próstata en Segundo y Tercer nivel de Atención. Guía Práctica Clínica GPC [Internet]. 2009;1-102. Disponible en: www.cenetec.salud.gob.mx.

13. Thomas AA, Pearce A, Sharp L, Gardiner RA, Chambers S, Aitken J, et al. Socioeconomic disadvantage but not remoteness affects short-term survival in prostate cancer: A population-based study using competing risks. Asia Pac J Clin Oncol. 2017;13(2):e31-40. doi: 10.1111/ajco.12570.

14. Yu XQ, Luo Q, Smith DP, O'Connell DL, Baade PD. Geographic variation in prostate cancer survival in New South Wales. Med J Aust. 2014;200(10):586-90. doi: 10.5694/mja13.11134.

15. Cayetano-Alcaraz AA, Ramírez-Rivera JA, Sotomayor-de-Zavaleta M, Castillejos-Molina RA, Gabilondo-Navarro F, Feria-Bernal G, et al. Características de los casos incidentes de cáncer de próstata en los últimos 5 años en un hospital de tercer nivel en México. Rev Mex Urol. 2016;76(2):76-80. doi: 10.1016/j.uromx.2015.11.007.

16. Migowski A, Silva GA. Survival of patients with clinically localized prostate cancer. Rev Saude Publica. 2010;44(2):344-52. doi: 10.1590/ s0034-89102010000200016.

17. Villegas C, Chacón J, Sánchez T. Sobrevida en cáncer de próstata de una población del centro de Colombia. Acta Med Colomb. 2015;40(2):101-8. doi: 10.36104/amc.2015.447.

18. Silveira F, Bandeira Lages R, Assuncao Costa U, Mendes Teles J, Campelo V. Survival of patients with prostate cancer. Rev Bras Promoc Saude. 2013;26(1):45-50.

19. Afshar N, English DR, Milne RL. Rural-urban residence and cancer survival in high-income countries: A systematic review. Cancer. 2019;135(3):2172-84. doi: 10.1002/cncr.32073.

20. Allemani C, Matsuda T, Carlo V Di, Harewood R, Matz M, Nikšić M, et al. Articles Global surveillance of trends in cancer survival 2000 - 14 (CONCORD-3): analysis of individual records for 37513025 patients diagnosed with one of 18 cancers from 322 population-based registries in 71 countries. Lancet. 2018;14(391):1023-75. doi: 10.1016/S01406736(17):33326-3.
21. Arias-Ortiz NE, de Vries E. Inequidades en salud y supervivencia al cáncer en Manizales, Colombia : un estudio de base poblacional. Colomb Med. 2018;49(6):63-72. doi: 10.25100/cm.v49i1.3629.

22. Hernández Morales M, Hernández Nieves A, García Grajales S, Ocampo Escobedo R, Luna Ruiz M, Ortiz Chacha C, et al. Mortalidad y factores sociales del cáncer de próstata para la gestión de políticas públicas. Veracruz, México. Investig Andin. 2017;19(35):163-83. doi: 10.33132/01248146.956.

23. Torres-Sánchez LE, Espinoza-Giacinto R, Rojas-Martínez R, Escamilla-Nuñez C, Vázquez-Salas RA, Campuzano JC, et al. Prostate cancer mortality according to marginalization status in Mexican states from 1980 to 2013. Salud Publica Mex. 2016;58(2):179-86.

24. Moghimi M, Shayestehpour M. Survival outcome in men with prostate cancer in Yazd Province, Central Iran, from 2001 to 2012. Asian Pacific J Cancer Biol. 2017;2(4):79-82. doi: 10.22034/APJCB.2017.2.4.79.

25. Hashibe M, Kirchhoff AC, Kepka D, Kim J, Millar M, Sweeney C, et al. Disparities in cancer survival and incidence by metropolitan versus rural residence in Utah. Cancer Med. 2018;7(4):1490-7. doi: 10.1002/cam4.1382.

26. Dasgupta P, Baade PD, Aitken JF, Ralph N, Chambers SK, Dunn J. Geographical variations in prostate cancer outcomes: A systematic review of international evidence. Front Oncol. 2019;9(2):238-73. doi: 10.3389/fonc.2019.00238.

27. Cortés A, Bravo LE, García LS, Collazos P. Incidencia, mortalidad y supervivencia por cáncer de próstata en Cali, Colombia, 1962-2012. Salud Publica Mex. 2014;56(5):457-64. doi: 10.21149/spm.v56i5.7371.

28. Eylert M, Bahl A, Hounsome L, Verne J, Jefferies E, Persad R. The impact of socio-economic deprivation on incidence, treatment and mortality from prostate cancer in England, 1990-2010. J Clin Urol. 2016;9(2):93-101. doi: 10.1177/2051415815594976.

29. Tomic K, Ventimiglia E, Robinson D, Häggström C, Lambe M, Stattin P. Socioeconomic status and diagnosis, treatment, and mortality in men with prostate cancer. Nationwide population-based study. Int J Cancer. 2018;142(12):2478-84. doi: 10.1002/ijc.31272.

30. Klein J, von dem Knesebeck O. Socioeconomic inequalities in prostate cancer survival: A review of the evidence and explanatory factors. Soc Sci Med. 2015;142:9-18. doi: 10.1016/j.socscimed.2015.07.006. 